\title{
SYNTHESIS OF 1-BENZYL-3-[4-(ARYL-1-PIPERAZINYL) CARBONYL]-1H-INDOLES. NOVEL LIGANDS WITH POTENTIAL D4 DOPAMINERGIC ACTIVITY
}

\author{
HERNÁN PESSOA-MAHANA ${ }^{1 *}$, IGNACIO CUEVAS M. ${ }^{1}$, C. DAVID PESSOA-MAHANA ${ }^{2}$. RAMIRO ARAYA- \\ MATURANA ${ }^{1}$ IRIUX ALMODOVAR FAJARDO, AND CLAUDIO SAITZ BARRÍA ${ }^{l}$
}

\begin{abstract}
${ }^{I}$ Department of Organic and Physical Chemistry, Faculty of Chemical and Pharmaceutical Sciences, University of Chile. Casilla 233. Santiago 1. Chile. ${ }^{2}$ Department of Pharmacy, Faculty of Chemistry, Pontificia Universidad Católica de Chile. Casilla 306. Santiago 22. Chile.
\end{abstract}

(Received: December 26, 2010 - Accepted: November 24, 2011)

\begin{abstract}
The synthesis of a series of functionalized 1-Benzyl-3-[4-Aryl-1-piperazin $\gamma 1]$ carbonyl-1H-Indoles $\mathbf{6}(\mathbf{a}-\mathbf{f})$, as a potential new class of bioactive ligands at $\mathrm{D}_{4}$ receptors is reported. The synthetic strategy took place through a five steps sequence to provide indole amides 6(a-f) in $75-92 \%$ yield.
\end{abstract}

Keywords : Indole, Arylpiperazines, dopaminergic activity.

\section{INTRODUCTION}

The indole ring system is present in many biologically active medicinal agents and natural products ${ }^{1-3}$. The first synthesis of substituted indoles was conducted by Fischer and Jourdan as early as 1883 , and since then the bicyclic heteroaromatic core has been the target of many synthetic approaches and reactivity studies. ${ }^{4-7}$ Indoles are also prominent structural elements in the neurotransmitter serotonin, the antiinflammatory drug indomethacin and other molecules showing promise in the treatment of cardiovascular disease, erectile dysfunction, cancer and neurological conditions such as the Alzheimer's disease $^{8-10}$

Interesting investigations on the role of dopaminergic system in the ethiology of neurological and psychiatric disorders such as Parkinson's disease and schizophrenia have been carried out in the last years ${ }^{11}$. In the course of these studies, and in an attempt to improve the activity of 3-(4-phenylpiperazin1-yl-methyl) indole (I) on $\mathrm{D}_{4}$ receptors (Fig.1). Troschütz ${ }^{12}$ and Gmeiner $^{13}$ have synthesized a series of new phenylpiperazinylmethyl indole derivatives. The 2-arylpiperazinylindolecarboxylates (II) displayed high affinity and great selectivity for the human dopamine $\mathrm{D}_{4}$ receptor over the other dopamine receptor subtypes. For instance the $\mathrm{Ki}$ value for compound (II), R=H was 1.9 $\mathrm{nM}$ (D4) over $\left(\mathrm{D}_{1}, \mathrm{D}_{2}\right.$ and $\left.\mathrm{D}_{3}>2000 \mathrm{nM}\right)$.

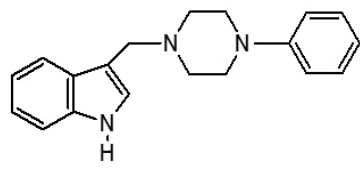

(I)

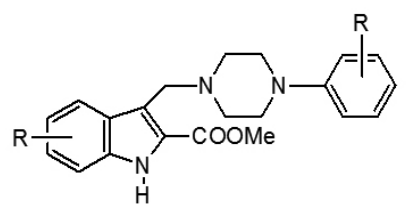

(II) $\mathrm{R}=\mathrm{H}, \mathrm{Cl}, \mathrm{F}, \mathrm{OMe}$
Considering the above results, and given our interest in the synthesis of neurobioactive indoles, we carry out the preparation of a new series of indolepiperazines (III) based on the incorporation of an amide function between the arylpiperazine ${ }^{14}$ and the indole framework. Selectivity studies respect to $\mathrm{D}_{4}$ binding affinity ${ }^{15}$, recognize the preference of compounds type (I) bearing substituents with a large negative region $(\mathrm{COOEt}, \mathrm{CN}, \mathrm{CHO}$, $\mathrm{CH}=\mathrm{NOH}$ ), which are naturally not well tolerated by the other D-receptors. In such sense, this new structural function may reinforce the stability of the ligand-receptor $\mathrm{D}_{4}$-complex, acting both as a hydrogen bond acceptor and providing the negative region required for $\mathrm{D}_{4}$ receptor selectivity as well.

To the best of our knowledge, these are the first examples of indoles amide connected to arylpiperazines, which will be pharmacologically evaluated in a near future. In this article we report the synthesis in good yield of a series of 1-benzyl-3-[4-Aryl-1-piperazin $\gamma 1]$ carbonyl-1 $H$-Indoles, with potential biological interest in $\mathrm{D}_{4}$ dopaminergic receptors.

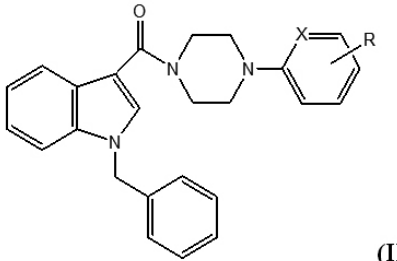

$\mathrm{X}=\mathrm{CH} ; \mathrm{R}=2-\mathrm{OMe}, 3-\mathrm{OMe}, 4-\mathrm{F}, 4-\mathrm{NO}_{2}, \mathrm{H}$.

$\mathrm{X}=\mathrm{N} ; \mathrm{R}=\mathrm{H}$

\section{RESULT AND DISCUSSION}

The N-benzyl-3-(4-aryl-1-piperazin $\gamma 1)$ carbonyl-1(H)-indoles (I) were obtained as follows: commercially available, indole (1) was subjected to a Vilsmeier-Haack formilation to provide $1 H$-Indole-3-carbaldehyde (2) in $85 \%$ yield. The aldehyde function was clearly detected in IR by its strong absorption band at $1634 \mathrm{~cm}^{-1}$ in accord with their aromatic and highly polar character, the ${ }^{1} \mathrm{H}-\mathrm{NMR}$ displayed a singlet signal at $\delta: 9.9 \mathrm{ppm}$. With the purpose to avoid secondary reactions such as N-oxidation, we decided to protect the indolic $\mathrm{N}-\mathrm{H}$, using benzyl bromide in dry DMF at $5{ }^{\circ} \mathrm{C}$, the reaction gave the N-Benzyl derivative (3) as a crystalline solid in $75 \%$ yield (Scheme 1 ). The electron-withdrawing effect of the formyl group on the indole ring, enhanced the acidity of the $\mathrm{NH}$, facilitating the proton abstraction and $\mathrm{N}$ - substitution.<smiles>c1ccc2[nH]ccc2c1</smiles>

(1)
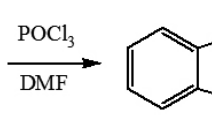

(2)

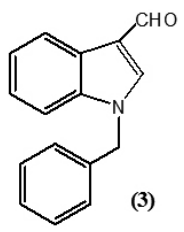

(3)
Scheme 1

Scheme 1. Preparation of 1-Benzyl-1H-indole-3-carbaldehyde(3).

Oxidation of (3) with $\mathrm{KMnO}_{4}$ in acetone-water (1:1) mixture, aforded the indole carboxylic acid derivative (4), (Scheme 2) which exhibited in IR the characteristic O-H absorption at $3420-2550 \mathrm{~cm}^{-1}$, along with a strong signal at $1655 \mathrm{~cm}^{-1}$ for the carboxylic function. At this point, it is interesting to comment that a first approach considered the preparation of 3-acyl halide indole (4-a), which would react in a second step with appropriate series of arylpiperazines. 
<smiles>O=C(O)c1cn(Cc2ccccc2)c2ccccc12</smiles>

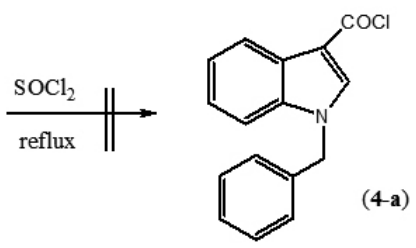

However, this reaction was unsuccessful giving a red-dark syrup displaying many products on thin layer chromatography, even under different experimental conditions. A probable explanation may arise of a policondensation reaction between the indolic rings under the acidic medium.

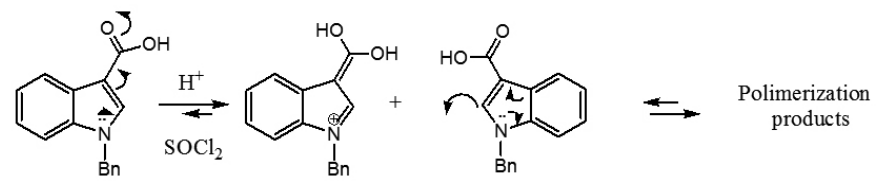

Thus, we decided to utilize the reaction of (4) with $\mathrm{N}, \mathrm{N}^{\prime}$ dicyclohexylcarbodiimide (DCC). Treatment of (4) with DCC in anhydrous $\mathrm{CH}_{2} \mathrm{Cl}_{2}$ gave the indole intermediate (5) in quantitative yield (Scheme 2), the ${ }^{1} \mathrm{H}$ NMR signals at $\delta: 0.8-2.0 \mathrm{ppm}$ for twenty protons, indicated the presence of the cyclohexyl rings. The signals at $\delta: 3.51 \mathrm{ppm}(\mathrm{m}, 1 \mathrm{H}, \mathbf{C H N H})$, and $\delta: 4.30$ $4.37 \mathrm{ppm}(\mathrm{m}, 1 \mathrm{H}, \mathbf{C H N}=)$ suported the methine protons.<smiles>O=Cc1cn(Cc2ccccc2)c2ccccc12</smiles>

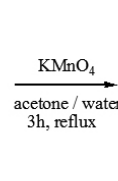<smiles>O=C(O)c1cn(Cc2ccccc2)c2ccccc12</smiles>

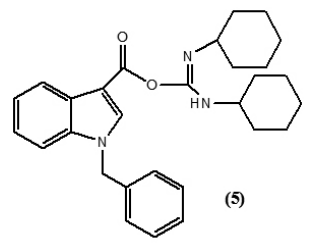

Scheme 2. Synthesis of 2-(1-Benzyl-1H-indole-3-carbonyl)-1,3dicyclohexyl-isourea.

Finally, the indole (5) was cleanly converted to the corresponding 1-benzyl-3-[4-aryl-1-piperazin $\gamma 1]$ carbonyl- $1 H$-indoles 6(a-f) in good yield 65$92 \%$ by reaction with a series of commercially available arylpiperazines.
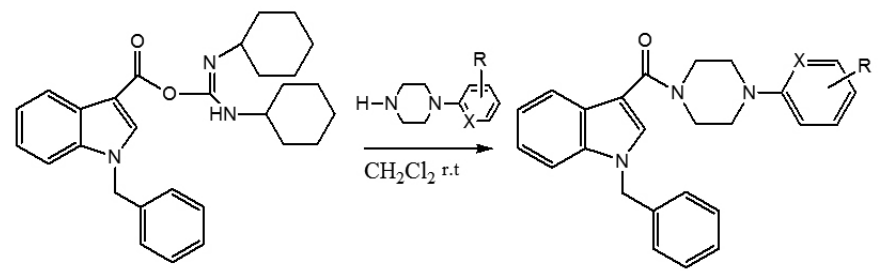

Formation of series $\mathbf{6 ( a - f )}$ was mainly supported in ${ }^{1} \mathrm{H}$ NMR by the presence of two bride singulets or two triplets assigned for the piperazine ring protons at $\delta: 3.57$ and $3.77 \mathbf{6 ( a )}, \delta: 3.0$ and $3.79 \mathbf{6 ( b )}, \delta: 3.17$ and $3.81 \mathbf{6 ( c )}$, $\delta: 3,21$ and $3,78 \mathbf{6 ( d )}, \delta: 3.58$ and $3.82 \mathbf{6 ( e )}$ and $\delta: 3.29$ and $3.88 \mathbf{6 ( f )}$ along with the aromatic proton signals. The $\mathrm{M}^{+}$obtained values in HRMS confirmed the proposed structures. (Table 1.)

Table 1. Physical constants for the 6(a-f) series.

\begin{tabular}{|c|c|c|c|}
\hline Products & $\begin{array}{c}\text { HRMS }\left(\mathrm{M}^{+}\right) \\
\text {Calculated / Experimental }\end{array}$ & m.p. ${ }^{\circ} \mathrm{C}$ & Yield \% \\
\hline 6-a & $396.19501 / 396.19505$. & $114-116$ & 65 \\
\hline 6-b & $425.21033 / 425.20978$. & $130-131$ & 92 \\
\hline 6-c & $413.19034 / 413.19025$ & $140-141$ & 78 \\
\hline 6-d & $425.21033 / 425.20922$ & $107-109$ & 82 \\
\hline 6-e & $440.18484 / 440.18490$ & $157-159$ & 87 \\
\hline 6-f & $395.19976 / 395.19940$ & $124-125$ & 89 \\
\hline
\end{tabular}

The mass spectrum analysis for indoles $\mathbf{6}(\mathbf{a}-\mathbf{f})$ showed the presence of a fragment $(\mathrm{m} / \mathrm{z})$ at 233.95 which can be explained assuming an $\alpha$-cleavage fragmentation pattern of an amide. The major fragment in this series involved a fragment $(\mathrm{m} / \mathrm{z})$ at 90.97 which may arise from a $\mathrm{N}$-debenzylation cleavage to yield the base peaks $(100 \%)$.

Finally, the N-debenzylation reaction of the indoles $\mathbf{6}(\mathbf{a}-\mathbf{f})$ will be carried out after the biological proofs, taking into account a possible favorable $\pi$ - $\pi$ interaction of the N-benzylic aromatic ring with aromatic aminoacidic residues with the receptor.

\section{CONCLUSION}

In conclusion new arylpiperazine indole derivatives have been synthesized in good yield, the utilized strategy provides an efficient method for the preparation of potentially bioactive ligands. Further efforts focused on the synthesis of new indole derivatives with potential biological relevance, along with neurobiological screening of the synthesized compounds are in progress.

\section{EXPERIMENTAL SECTION}

Melting points were determined on a hot-stage apparatus and are uncorrected. The IR spectra were recorded, on a FT-IR Bruker IFS 55 spectrophotometer for $\mathrm{KBr}$ disc and wave numbers are reported in $\mathrm{cm}^{-1}$. The ${ }^{1} \mathrm{H}$ NMR and ${ }^{13} \mathrm{C}$ NMR spectra were performed on a Bruker DRX-300 spectrometer (300 and $75 \mathrm{MHz}$ ) in deuterochloroform, or DMSO-d $\mathrm{d}_{6}$. Chemical shifts were recorded in ppm $(\delta)$ relative to TMS as an internal standard. $J$ values are given in Hz. Microanalyses were carried out on a Fisons EA 1108 analizer. High resolution mass spectrum were recorded on a Thermo Finnigan model MAT 95XP Mass Spectrometer. Silica gel Merck 60 (70-230mesh) and DC- alufolien $60 \mathrm{~F}_{254}$ were used for column and TLC chromatography respectively.

\section{1 $1 H$-Indole-3-carbaldehyde (2).}

To a solution of indole (1) at $0{ }^{\circ} \mathrm{C},(500 \mathrm{mg}, 4.27 \mathrm{mmol})$ in DMF $(2,5 \mathrm{~mL})$, was added drop by drop a solution of $\mathrm{POCl}_{3}(0,4 \mathrm{~mL}, 4.36 \mathrm{mmoles})$ in $\mathrm{DMF}(2,5$ $\mathrm{mL}$ ) recently prepared $(1 \mathrm{~h})$ and the mixture stirred for $30 \mathrm{~min}$. After stirring the mixture was poured onto a water-ice mixture and basified with $\mathrm{NaOH}(0.5 \mathrm{M})$ to $\mathrm{pH}: 12$. The obtained white-yellow precipitate was filtered off and dried to yield $526 \mathrm{mg}, 85 \%)$ of pure indole (2). $\mathrm{mp} 181-182^{\circ} \mathrm{C} ; \mathrm{IR}_{\mathrm{rax}}\left(\mathrm{cm}^{-1}\right): 3168(\mathrm{~N}-$ $\mathrm{H}), 1634(\mathrm{C}=\mathrm{O}), 1576(\mathrm{C}=\mathrm{C}) ;{ }^{1} \mathrm{H}$ NMR $\left(300 \mathrm{MHz}, \mathrm{CDCl}^{\mathrm{vmax}}\right): 7.21(\mathrm{~m}, 2 \mathrm{H}, 5-\mathrm{H}$ and 6-H), 7,49 (dd, $\left.1 \mathrm{H}, 7-\mathrm{H}, J=7.1 \mathrm{~Hz}, J_{\mathrm{m}}=1.5 \mathrm{~Hz}\right), 8.1(\mathrm{dd}, 1 \mathrm{H}, 4-\mathrm{H}, J=6.7$ $\mathrm{Hz}, J=1.9 \mathrm{~Hz}), 9.9(\mathrm{~s}, 1 \mathrm{H}, \mathrm{CHO}), 12.1(\mathrm{~s}, 1 \mathrm{~m}, \mathrm{NH}) .{ }^{13} \mathrm{C} \mathrm{NMR}(75 \mathrm{MHz}, \stackrel{\mathrm{C} D C l}{ })$ : 112.4, 118.2, 121.1, 122.1, 123.4, 124.1, 137.0, 138.4, 184.9. HRMS (EI) Calcd for $\mathrm{C}_{9} \mathrm{H}_{7} \mathrm{NO},:$ : 145.05276. Found (M-1) : 144.0445 .

\section{1-Benzyl-1H-indole-3-carbaldehyde. (3).}

To a solution of indole (2) $(500 \mathrm{mg}, 3.44 \mathrm{mmol})$ in dry DMF (10 ml) NaH (123 mg, $5.16 \mathrm{mmol}, 60 \%$ suspension in mineral oil) was added slowly. The reaction mixture was stirred and cooled to $5{ }^{\circ} \mathrm{C}$, then benzyl bromide $(816 \mathrm{mg}$, $4.8 \mathrm{mmol}$ ) was added dropwise.

After stirring for $30 \mathrm{~min}$, the mixture was poured onto a water-ice mixture and a white-pink precipitated was formed to yield pure indole (3) $607 \mathrm{mg}$ , $75 \%$ ). mp. $94-95^{\circ} \mathrm{C}$. IR ( $\left.\mathrm{cm}^{-1}\right) .3108$ (C-H Arom.), 2815 (C-H aliph.), 1661 $(\mathrm{C}=\mathrm{O}), 1536(\mathrm{C}=\mathrm{C}) .{ }^{\mathrm{vmax}}{ }^{\mathrm{H}} \mathrm{H}$ NMR $\left(300 \mathrm{MHz}, \mathrm{CDCl}_{3}\right): 5.3\left(\mathrm{~s}, 2 \mathrm{H}, \mathrm{Ar}-\mathbf{C H}_{2}-\right)$, 7.14-7.35 (m,8H, 5-H, 6-H, 7-H, and Ar-CH $), 7.7(\mathrm{~s}, 1 \mathrm{H}, 2-\mathrm{H}), 8.3(\mathrm{~m}, 1 \mathrm{H}$, 4-H), 9.97(s,1H,CHO). ${ }^{13} \mathrm{C}$ NMR (75 MHz, $\left.\mathrm{CDCl}_{3}\right): 50.4,109.9,118.0,121.7$, $122.6,123.7,125.0,126.8(2 \mathrm{C}), 127.8,128.7(2 \mathrm{C}), 134.9,137.0,138.1,184.2$. HRMS (EI) Calcd for $\mathrm{C}_{16} \mathrm{H}_{13} \mathrm{NO},\left(\mathrm{M}^{+}\right): 235.09971$. Found : 235.09946.

\section{1-Benzyl-1H-indole-3-carboxylic acid (4).}

To a solution of indole aldehyde (3) $(420 \mathrm{mg}, 1.78 \mathrm{mmol})$ in acetone -water mixture $(80 \mathrm{~mL}, 1: 1 \mathrm{v} / \mathrm{v})$ was added $\mathrm{KMnO}_{4}(1.120 \mathrm{mg}, 7.12 \mathrm{mmol})$. The mixture was stirred for $3 \mathrm{~h}$, filtered on celite and concetrated in vacuo to remove the organic solvent. The resulting aqueous solution was cooled and acidified with $\mathrm{HCl}$ (concd.) to afford a white precipitate, which was collected by filtration and dried to provide pure carboxylic acid (4) (358 mg, 80\%). mp :183-184 ${ }^{\circ} \mathrm{C}$. IR $\quad\left(\mathrm{cm}^{-1}\right)$ : 3420-2550 (O-H), 3031 (C-H Arom.), 2931(C-H aliph.), $1655(\mathrm{C}=\mathrm{O}), 1576(\mathrm{C}=\mathrm{C}), 1220(\mathrm{C}-\mathrm{O}) .{ }^{1} \mathrm{H}$ NMR (300 MHz, $\left.\mathrm{CDCl}_{3}\right)$ : $5.43\left(\mathrm{~s}, 2 \mathrm{H}, \mathrm{Ar}-\mathrm{CH}_{-}\right), 7.1-7.29\left(\mathrm{~m}, 7 \mathrm{H}, 5-\mathrm{H}, 6-\mathrm{H}\right.$, and $\left.\mathrm{Ar}-\mathrm{CH}_{2}\right), 7.47(\mathrm{~m}$, $1 \mathrm{H}, 4-\mathrm{H}), 8.00(\mathrm{~m}, 1 \mathrm{H}, 7-\mathrm{H}), 8.18(\mathrm{~s}, 1 \mathrm{H}, 2-\mathrm{H}), 12.1$ (br.s., $1 \mathrm{H}, \mathrm{COOH}) .{ }^{13} \mathrm{C}$ NMR (75 MHz, $\left.\mathrm{CDCl}_{3}\right)$ : 50.0, 107.4, 111.6, 121.4, 121.9, 122.8, 127.1, 127.8 $(2 \mathrm{H}), 128.1,129.1(2 \mathrm{H}), 135.9,136.8,137.6,166.0$. HRMS (EI) Calcd for $\mathrm{C}_{16} \mathrm{H}_{13} \mathrm{NO}_{2}\left(\mathrm{M}^{+}\right): 251.09463$. Found : 251.09446 . 
2-(1-Benzyl-1H-indole-3-carbonyl)-1,3-dicyclohexyl-isourea (5).

To a solution of 3-indole carboxylic acid (4) $(500 \mathrm{mg}, 1.99 \mathrm{mmol})$ in $\mathrm{CH}_{2} \mathrm{Cl}_{2}(20 \mathrm{~mL})$, was added N,N'-dicyclohexylcarbodiimide $(490 \mathrm{mg}, 2.38$ mmoles) and 4-dimethylaminopyridine ( $290 \mathrm{mg} ; 2.38 \mathrm{mmol})$. The mixture was stirred at room temperature for $90 \mathrm{~min}$. The solvent was removed in vacuo and the residue purified by column chromatography (EtOAc: Hexane 1:1) to afford (5) $\left(880 \mathrm{mg}\right.$, quantitative yield). $\mathrm{mp}: 169-171^{\circ} \mathrm{C} . \mathrm{IR}_{\mathrm{vmax}}\left(\mathrm{cm}^{-1}\right): 3327(\mathrm{~N}-\mathrm{H})$, 3032 (C-H Arom.), 2927(C-H aliph.), 1752(C=O), $1696(\mathrm{C}=\mathrm{N}), 1575(\mathrm{C}=\mathrm{C})$. ${ }^{1} \mathrm{H}$ NMR : 0.8-2.2 (m, $\left.20 \mathrm{H}\right), 3.43-3.51(\mathrm{~m}, 1 \mathrm{H}, \mathbf{C H N H}), 4.30-4.37(\mathrm{~m}, 1 \mathrm{H}$, $\mathbf{C H N}=), 5.29\left(\mathrm{~s}, 2 \mathrm{H}, \mathbf{C H}_{2}-\mathrm{C}_{6} \mathrm{H}_{5}\right), 6.04(\mathrm{~d}, 1 \mathrm{H}, \mathrm{NH}, J=5.7 \mathrm{~Hz}), 7.59(\mathrm{~s}, 1 \mathrm{H}, 2-\mathrm{H})$, $8.06(\mathrm{dd}, 1 \mathrm{H}, 4-\mathrm{H}, J o=4.8 \mathrm{~Hz}, J m=2.1 \mathrm{~Hz}), 7.14-7.32\left(\mathrm{~m}, 8 \mathrm{H}, \mathrm{CH}_{2}-\mathbf{A r}\right.$ and $5-\mathrm{H}, 6-\mathrm{H}, 7-\mathrm{H}) .{ }^{13} \mathrm{C}$ NMR $(75 \mathrm{MHz}): 24.6,25.4,25.5(2 \mathrm{C}), 26.4(2 \mathrm{C}), 31.2(2 \mathrm{C})$, $32.5(2 \mathrm{C}), 49.7,50.6,57.1,110.2,111.9,121.7,121.9,123.2,127.1(2 \mathrm{C}), 127.4$, 128.2(2C), 129.0, 131.1, 136.0, 136.5, 155.3, 166.8. HRMS (EI) Calcd for $\mathrm{C}_{29} \mathrm{H}_{35} \mathrm{~N}_{3} \mathrm{O}_{2},\left(\mathrm{M}^{+}\right): 457.27293$. Found: 457.27474.

General Procedure for the Synthesis of 1-Benzyl-3-[4-(aryl-1piperazinyl) carbonyl]-1H-Indoles $6(\mathrm{a}-\mathrm{f})$.

1-Benzyl-3-[4-(2-pyridinyl-1-piperazinyl)carbonyl]-1 $H$-Indole (6-a).

To a solution of 1-Pyridin-2-yl-piperazine $(220 \mathrm{mg}, 1.35 \mathrm{mmol})$ in $\mathrm{CH}_{2} \mathrm{Cl}_{2}$ $(30 \mathrm{~mL})$ was added 2-(1-Benzyl-1H-indole-3-carbonyl)-1,3-dicyclohexylisourea (5), (618 mg, $1.35 \mathrm{mmol}$ ) and the mixture stirred at room temperature for $1 \mathrm{~h}$. The crude residue was concentrated in vacuo, and purified by column chromatography (EtOAc/ Hexane 1:1) to give pure 6(a) (386 mg, 65\%). mp : 114-116 ${ }^{\circ} \mathrm{C}$. IR $\left(\mathrm{cm}^{-1}\right): 3027$ (C-H Arom.), $1619(\mathrm{C}=\mathrm{O}), 1542(\mathrm{C}=\mathrm{C}) .{ }^{1} \mathrm{H}$ NMR (300 MHz, DMSO-d $)$ ): 3.57 (b.s. 4H, 2'-H, 6'-H), 3.77 (b.s., 4H, 3'-H, 5 '-H), 5.40 (s,2H, CH -Ar), 6.59(t,1H, 5"'-H, $J=5.9 \mathrm{~Hz}) 6.71(\mathrm{~d}, 1 \mathrm{H}, 3$ '”'-H, $J=8.4 \mathrm{~Hz}), 7.05-7.25^{2}\left(\mathrm{~m}, 7 \mathrm{H}, 5-\mathrm{H}, 6-\mathrm{H}\right.$ and $\left.\mathbf{A r}-\mathrm{CH}_{2}\right), 7.37(\mathrm{~m}, 1 \mathrm{H}, 7-\mathrm{H}), 7.46$ $(\mathrm{t}, 1 \mathrm{H}, 4$ "'- $\mathrm{H}, J=7.8 \mathrm{~Hz}), 7.74(\mathrm{~m}, 2 \mathrm{H}, 4-\mathrm{H}$ y $2-\mathrm{H}), 8.10(\mathrm{~d}, 1 \mathrm{H}, 6$ "'- $-\mathrm{H}, J=$ $4.2 \mathrm{~Hz}) \cdot{ }^{13} \mathrm{C}$ RMN ( $75 \mathrm{MHz}$, DMSO-d $): 44.8(2 \mathrm{C}), 45.4,50.1(2 \mathrm{C}), 107.5$ , 109.9 , 110.8, 113.6, 120.9, 121.0, 122.6, 127.0, 127.3 (2C), 127.9, 128,.8, $128.9(2 \mathrm{C}), 131.5,136.0,137.7,147.9,159.2,166.0$. HRMS (EI) Calcd for $\mathrm{C}_{25} \mathrm{H}_{24} \mathrm{~N}_{4} \mathrm{O}\left(\mathrm{M}^{+}\right): 396.19501$. Found : 396.19505 .

1-Benzyl-3-\{[4-(2-methoxyphenyl)-1-piperazinyl]carbonyl\}-1HIndole.(6-b)

Prepared from 1-(2-methoxyphenyl)-piperazine (490 mg, $2.55 \mathrm{mmol})$ and 2-(1-Benzyl-1H-indole-3-carbonyl)-1,3-dicyclohexyl-isourea (5), (1167 mg, $2.55 \mathrm{mmol}$ ), to give crude $\mathbf{6}(\mathbf{b})$ in quantitative yield. The residue was purified by column chromatography (EtOAc/ Hexane 1:1) to yield 6(b) (307 mg, 92\%). mp : $130-131{ }^{\circ} \mathrm{C}$. IR $\left(\mathrm{cm}^{-1}\right)$ : $3015(\mathrm{C}-\mathrm{H}$ Arom. $), 1610(\mathrm{C}=\mathrm{O}), 1541(\mathrm{C}=\mathrm{C})$. ${ }^{1} \mathrm{H}$ NMR (300 MHz, DMSO-d d $_{6}$ ) : 3.0 (b.s., $4 \mathrm{H}, 3$ '-H and 5'-H), 3.79 (b.s., $7 \mathrm{H}$, 2'-H, 6'-H and $\mathrm{OMe}$ ), 5.47 (s,2H, CH-Ar), 6.92(m,4H, 3'- $-\mathrm{H}, 4$ ''- $\mathrm{H}, 5$ '”and 6"-H), 7.12-7.37 (m,7H, 5-H,6-H and 5x CH $-\mathbf{A r}), 7.49(\mathrm{~m}, 1 \mathrm{H}, 7-\mathrm{H}), 7.76$ $(\mathrm{m}, 1 \mathrm{H}, 4-\mathrm{H}), 7.98(\mathrm{~s}, 1 \mathrm{H}, 2-\mathrm{H}) \cdot{ }^{13} \mathrm{C}$ NMR $\left(75 \mathrm{MHz}, \mathrm{DMSO}-d_{6}\right): 45.5(2 \mathrm{C})$, $49.8,51.1(2 \mathrm{C}), 55.8,109.8,111.3,112.3,118.8,121.0,121.1,121.3,122.6$, 123.3, 127.2, 127.7(2C), 128.0, 129.1(2C), 132.0, 136.0, 138.0, 141.3, 152.5, 165.4. HRMS (EI) Calcd for $\mathrm{C}_{27} \mathrm{H}_{27} \mathrm{~N}_{3} \mathrm{O}\left(\mathrm{M}^{+}\right): 425.21033$. Found : 425.20978 (6-c)

1-Benzyl-3-\{[4-(4-fluorophenyl)-1-piperazinyl]carbonyl\}-1 $H$-Indole.

Prepared from 1-(4-fluorophenyl)-piperazine (330 mg, $1.83 \mathrm{mmol}$ ), and 2-(1-Benzyl-1H-indole-3-carbonyl)-1,3-dicyclohexyl-isourea (5), $(836$ $\mathrm{mg}, 1.83 \mathrm{mmol}$ ) to give crude $\mathbf{6}(\mathrm{c})$ in quantitative yield. Purified by column chromatography (EtOAc/ Hexane 1:1), to yield (589 mg, 78\%). mp : 140$141{ }^{\circ} \mathrm{C} . \mathrm{IR}_{\mathrm{rmy}}\left(\mathrm{cm}^{-1}\right)$ : 3058 (C-H Arom.), 2927 (C-H aliph.), $1625(\mathrm{C}=\mathrm{O})$, $1580(\mathrm{C}=\mathrm{C}) .{ }^{\mathrm{v}} \mathrm{H}$ NMR $\left(300 \mathrm{MHz}, \mathrm{DMSO}-d_{6}\right): 3.17\left(\mathrm{~m}, 4 \mathrm{H}, 3^{\prime}-\mathrm{H}\right.$ and $\left.5^{\prime}-\mathrm{H}\right)$, 3.81(m,4H, 2'-H, 6'-H), 5.51 (s,2H, CH $\left.\mathbf{H}_{2}-\mathrm{Ar}\right), 6.94-7.35(\mathrm{~m}, 11 \mathrm{H}, 5-\mathrm{H}, 6-\mathrm{H}$, 2"-H, 3"-H, 5"-H 6"-H and $\left.\mathrm{CH}_{2}-\mathrm{Ar}\right), 7.54\left(\mathrm{~d}, 1 \mathrm{H}, 7-\mathrm{H}, \mathrm{JO}_{0}=7.3 \mathrm{~Hz}\right), 7.77$ $\left(\mathrm{dd}, 1 \mathrm{H}, 4-\mathrm{H}, J o=6,5 \mathrm{~Hz}, J m=1.5^{2} \mathrm{~Hz}\right), 8.01(\mathrm{~s}, 1 \mathrm{H}, 2-\mathrm{H}) .{ }^{13} \mathrm{C}$ NMR $(75 \mathrm{MHz}$, DMSO- $d$ ) : $45.0(2 \mathrm{C}), 49.8,50.0(2 \mathrm{C}), 109.8,111.3,115.8\left(\mathrm{~d}, 2 \mathrm{C},{ }^{2} J=22 \mathrm{~Hz}\right)$, $118.2\left(\mathrm{~d}, 2 \mathrm{C},{ }^{3} \mathrm{~J}=7.6 \mathrm{~Hz}\right), 121.0,121.1,122.6,127.1,127.7(2 \mathrm{C}), 128.0,129.1$ (2C), 132.1, 136.0, 138.0, $148.3\left(\mathrm{~d},{ }^{4} J=2.0 \mathrm{~Hz}\right), 156.8\left(\mathrm{~d},{ }^{1} J=227 \mathrm{~Hz}\right), 165.5$. HRMS (EI) Calcd for $\mathrm{C}_{26} \mathrm{H}_{24} \mathrm{FN}_{3} \mathrm{O}\left(\mathrm{M}^{+}\right): 413.19034$. Found : 413.19025.

1-Benzyl-3-\{[4-(3-methoxyphenyl)-1-piperazinyl]carbonyl\}-1HIndole. (6-d)

Prepared from 1-(3-methoxy-phenyl)-piperazine $(420 \mathrm{mg}, 2.18 \mathrm{mmol})$ and 2-(1-Benzyl-1 $H$-indole-3-carbonyl)-1,3-dicyclohexyl-isourea (5), (1000 $\mathrm{mg}, 2.18 \mathrm{mmol}$ ), to give crude $\mathbf{6}(\mathbf{d})$ in quantitative yield. Purified by column chromatography (EtOAc/ Hexane 1:1) to yield (760 mg, 82\%). mp : 107-109 ${ }^{\circ} \mathrm{C} . \mathrm{IR}_{\text {vmax }}\left(\mathrm{cm}^{-1}\right): 3032$ (C-H arom.), 2927 (C-H aliph.), $1626(\mathrm{C}=\mathrm{O}), 1578$
$(\mathrm{C}=\mathrm{C}) .{ }^{1} \mathrm{H}$ NMR $\left(300 \mathrm{MHz}\right.$, DMSO- $\left.d_{6}\right): 3.21\left(\mathrm{~m}, 4 \mathrm{H}, 3^{\prime}-\mathrm{H}\right.$ and 5 ' $\left.-\mathrm{H}\right), 3.72$ (s,3H, OMe), 3.78 (m,4H, 2'-H and 5'-H), 6.40(d,1H, 6'- $-\mathrm{H}, J=8.0 \mathrm{~Hz}), 6.50$ (s,1H, 2"-H), 6.56 (d,1H, 4"-H), 7.05-7.22 (m,3H, 5"'-H and 2x Ar-CH $), 7.25$ $(\mathrm{m}, 5 \mathrm{H}, 5-\mathrm{H}, 6-\mathrm{H}$ and $3 \mathrm{x}$ Ar- $\mathrm{CH}), 7.51(\mathrm{~d}, 1 \mathrm{H}, 7-\mathrm{H}, J=7.7 \mathrm{~Hz}), 7.74(\mathrm{~d}, 1 \mathrm{H}$, $4-\mathrm{H}, J=7.3 \mathrm{~Hz}), 7.98(\mathrm{~s}, 1 \mathrm{H}, 2-\mathrm{H}) .{ }^{13} \mathrm{C}$ NMR ( $\left.75 \mathrm{MHz}, \mathrm{DMSO}-d_{6}\right): 45.0(2 \mathrm{C})$, $49.2,49.8(2 \mathrm{C}), 55.3,102.4,105.0,108.9,109.7,111.3,121.1(2 \mathrm{C}), 122.6$, 127.2, 127.7 (2C), 128.0, 129.1(2C), 130.1, 132.0, 136.0, 138.0, 152.7, 160.7, 165.6. HRMS (EI) Calcd for $\mathrm{C}_{27} \mathrm{H}_{27} \mathrm{~N}_{3} \mathrm{O}_{2}\left(\mathrm{M}^{+}\right): 425.21033$. Found : 425.20922. (6-e)

1-Benzyl-3-\{[4-(4-nitrophenyl)-1-piperazinyl]carbonyl\}- $1 H$-Indole.

Prepared from 1-(4-nitrophenyl)-piperazine (468 mg; 2.1mmol) and 2-(1-Benzyl-1H-indole-3-carbonyl)-1,3-dicyclohexyl-isourea (5), (961 mg, $2.1 \mathrm{mmol}$ ), stirred for $6 \mathrm{~h}$ to give crude ( 6-e) in quantitative yield. Purified by column chromatography (EtOAc/ Hexane 1:3) to yield. 6-e (988 mg, $87 \%$ ). $\mathrm{mp}: 157-159^{\circ} \mathrm{C} . \mathrm{IR}_{\text {vmax }}\left(\mathrm{cm}^{-1}\right)$ : 3029 (C-H arom.), 2927 (C-H aliph.), 1615 $(\mathrm{C}=\mathrm{O}), 1592\left(\mathrm{NO}_{2}\right.$ asym. $), 1328$ ( $\mathrm{NO}_{2}$ sym.). ${ }^{1} \mathrm{H}$ NMR (300 MHz, DMSO- $\left.d_{6}\right)$ : $3.58\left(\mathrm{t}, 4 \mathrm{H}, 3^{\prime}-\mathrm{H}^{2}\right.$ and $\left.5^{\prime}-\mathrm{H}, J=3.6 \mathrm{~Hz}\right), 3.82\left(\mathrm{t}, 4 \mathrm{H}, 2^{\prime}-\mathrm{H}^{\prime}\right.$ and $6^{\prime}-\mathrm{H}, J=3.6$ $\mathrm{Hz}), 5.49$ (s,2H, CH-Ar), 7.00 (d,2H, 2"-H and 6"-H, $J=9.4 \mathrm{~Hz}), 7.10-7.38$ $\left(\mathrm{m}, 7 \mathrm{H}, \mathbf{A r}-\mathrm{CH}_{2}\right.$ and 5-H, 6-H), $7.52(\mathrm{~d}, 1 \mathrm{H}, 7-\mathrm{H}, J=7.2 \mathrm{~Hz}), 7.81(\mathrm{dd}, 1 \mathrm{H}, 4-\mathrm{H}$, $J o=7.7$ and $J m=1.5 \mathrm{~Hz}), 8.02(\mathrm{~s}, 1 \mathrm{H}, 2-\mathrm{H}), 8.08(\mathrm{~d}, 2 \mathrm{H}, 3$ " $-\mathrm{H}$ and 5 "'- $\mathrm{H}, J=$ $9.4 \mathrm{~Hz}) .{ }^{13} \mathrm{C}$ NMR ( $75 \mathrm{MHz}$, DMSO- $\left.d_{6}\right): 44.5(2 \mathrm{C}), 46.7,49.9$ (2C), 109.5, 111.3, 112.9 (2C), 122.7, 121.1, 121.2, 126.2 (2C), 127.3, 127.7 (2C), 128.0, 129.1 (2C), 132.2, 136.0, 137.4, 138.0, 154.9, 165.7. HRMS (EI) Calcd for $\mathrm{C}_{26} \mathrm{H}_{24} \mathrm{~N}_{4} \mathrm{O}_{3}\left(\mathrm{M}^{+}\right): 440.18484$. Found : 440.18490 .

\section{1 -Benzyl-3-[(4-phenyl-1-piperazinyl)carbonyl]-1 $H$-Indole. (6-f)}

Prepared from 1-Phenyl-piperazine $(177 \mathrm{mg}, 1.09 \mathrm{mmol})$ and 2-(1-Benzyl$1 H$-indole-3-carbonyl)-1,3-dicyclohexyl-isourea (5), (499 mg, 1.09 mmoles ), stirred for $1.5 \mathrm{~h}$ to give crude ( 6-f) in quantitative yield. Purified by column chromatography (EtOAc / Hexane 1:1) to yield ( 6-f) (384 mg, 89\%). mp : $124-125^{\circ} \mathrm{C} . \mathrm{IR}_{\mathrm{vmax}}\left(\mathrm{cm}^{-1}\right): 3033(\mathrm{C}-\mathrm{H}$ arom. $), 2927(\mathrm{C}-\mathrm{H}$ aliph.), $1626(\mathrm{C}=\mathrm{O})$, $1576(\mathrm{C}=\mathrm{C}$ Arom $) .{ }^{1} \mathrm{H}$ NMR $(300 \mathrm{MHz}, \mathrm{DMSO}-d): 3.29\left(\mathrm{t}, 4 \mathrm{H}, 3^{\prime}-\mathrm{H}\right.$ and $5{ }^{\prime}-\mathrm{H}$, $J=4.9), 3.88\left(\mathrm{t}, 4 \mathrm{H}, 2^{\prime}-\mathrm{H}\right.$ and $\left.6^{\prime}-\mathrm{H}, J=4,9 \mathrm{~Hz}\right), 5.38\left(\mathrm{~s}, 2 \mathrm{H}, \mathrm{CH}_{2}-\mathrm{Ar}\right), 6.86(\mathrm{t}, 1 \mathrm{H}$, 4"-H, $J=7.3 \mathrm{~Hz}), 6.94(\mathrm{~d}, 2 \mathrm{H}, 2$ "- $\mathrm{H}$, and 6"- $\mathrm{H}, J=7.9 \mathrm{~Hz}), 7.15-7.38(\mathrm{~m}, 10 \mathrm{H}$, 3"-H, 5"-H, Ar-CH, 5-H, 6-H and 7-H ), $7.58(\mathrm{~s}, 1 \mathrm{H}, 2-\mathrm{H}), 7.76(\mathrm{~m}, 1 \mathrm{H}, 4-\mathrm{H})$. ${ }^{13} \mathrm{C}$ NMR ( $75 \mathrm{MHz}$, DMSO- $d_{6}$ ) : 44.9 (2C), 49.4, 50.0 (2C), 110.1, 110.2, 116.2 (2C), 119.9, 120.4, 120.8, 122.4, 126.3, 126.8 (2C), 127.6, $128.6(2 \mathrm{C})$, 128.9 (2C), 130.6, 135.7, 136.3, 150.8, 165.9 HRMS (EI) Calcd for $\mathrm{C}_{26} \mathrm{H}_{25} \mathrm{~N}_{3} \mathrm{O}$, $\left(\mathrm{M}^{+}\right)$: 395.19976. Found : 395.19940.

\section{ACKNOWLEDGEMENTS}

We acknowledge with thanks to PROYECTO FONDECYT 1090169 for the financial support.

\section{REFERENCES}

1. I.Borza, S. Kolok, A. Gere, E. Àgai-Csongor, B.Ágai, G. Tárkányi, C. Horváth, G. Barta-Szalai, E.Bozó, C. Kiss, A.Bielik, J. Nagy, S.Farkasa and G. Dománya, Bioorg. Med. Chem. Lett. 13, 3859-3861, (2003).

2. S. Pedpradab, R.A. Edrada, R. Ebel, V. Wray, and P. Proksch. J. Nat. Prod. 67, 2113-2116, (2004).

3. T. Heinrich, H. Bottcher, G. D. Bartoszyk, H. E. Greiner; Ch. A. Seyfried; and Ch. van Ámsterdam. J. Med. Chem. 47, 4677-4683, (2004).

4. T. Opatz and D. Ferenc, Org. Lett. 8, 20,4473-4475, (2006).

5. G. W. Gribble, J. Chem. Soc., Perkin Trans. 1. 1045-1075 (2000).

6. G. R. Humphrey; J. T. Kuethe, Chem. Rev., 106, 2875-2911, (2006).

7. H. Pessoa-Mahana, M. González, M. González, C.David.Pessoa-Mahana, R. Araya-Maturana, N. Ron, C.Saitz. Arkivoc (xi), 316-325, (2009).

8. I. Borza; E.A. Bozo'; G. Barta-Szalai, C. Kiss, G. Tárkányi, A. Demeter, T. Gáti, V. Háda, S. Kolok, A. Gere, L. Fodor, J. Nagy, K. Galgóczy, I. Magdo', B. A. Agai, J. F. F. Bertha, G. M. Keseru, C. Horváth, S. Farkas, I. Greiner, and G. Domány. J. Med. Chem. 50, 901-914, (2007).

9. T. Heinrich, H. Bottcher, K. Schiemann, G. Hölzemann, M. Schwarz, G. D. Bartoszyk, Ch. van Ámsterdam, H. E. Greiner, and Ch. A. Seyfried. Bioorg. Med. Chem. 12, 4843-4852, (2004).

10. P. Muñoz-Ruiz, L. Rubio, E. García-Palomero, I. Dorronsoro, M. del Monte-Millán, R. Valenzuela, P. Usán, C. de Austria, M. Bartolini, V. Andrisano, A. Bidon-Chanal, M. Orozco, F. J. Luque, M. Medina, and A. Martínez.. J. Med. Chem. 48, 7223-7233, (2005).

11. S. Vangveravong, E. McElveen, M. Taylor, J. Xu, Z. Tu, R. R. Luedtkeb and R. H. Macha, Bioorg. Med. Chem. 14, 815-825, (2006). 
12. A. Moll, H. Hübner, P. Gmeiner and R. Troschütz. Bioorg. Med. Chem. 10, 1671-1679, (2002).

13. H. Hübner, J. Kraxner, and P. Gmeiner, J. Med. Chem. 43, 4563-4569, (2000).
14. H. Pessoa-Mahana, C. González L., R. Araya- Maturana, Claudio Saitz B., C. David Pessoa-Mahana. J. Chil. Chem. Soc., 54, 2, 147-150 (2009). 15. S.Löber, Hübner, P. Gmeiner, Bioorg. Med. Chem. Lett. 9, 97-102, (1999). 